\title{
Neurologic Manifestations of Systemic Disease: Sleep Disorders
}

Eric M. Davis, $M D^{1, *}$

Chintan Ramani, MBBS ${ }^{1}$

Mark Quigg, MD, MSC ${ }^{2}$

\author{
Address \\ ${ }^{*}, 1$ Division of Pulmonary and Critical Care, Department of Medicine, University of \\ Virginia, Charlottesville, VA, USA \\ Email: emd9b@virginia.edu \\ ${ }^{2}$ Department of Neurology, University of Virginia, Charlottesville, VA, USA
}

Published online: 6 August 2020

(C) Springer Science+Business Media, LLC, part of Springer Nature 2020

This article is part of the Topical Collection on Neurologic Manifestations of Systemic Disease

Keywords Sleep disorders - Sleep manifestations of systemic diseases - Sleep impacts on health - Sleep apnea . Insomnia

\begin{abstract}
Purpose of review Sleep is intimately involved in overall health and wellbeing. We provide a comprehensive report on the interplay between systemic diseases and sleep to optimize the outcomes of systemic disorders.

Recent findings Spanning the categories of endocrinologic disorders, metabolic/toxic disturbances, renal, cardiovascular, pulmonary, gastrointestinal, infectious diseases, autoimmune disorders, malignancy, and critical illness, the review highlights the prevalent coexisting pathology of sleep across the spectrum of systemic disorders. Although it is rare that treating a sleep symptom can cure disease, attention to sleep may improve quality of life and may mitigate or improve the underlying disorder. Recent controversies in assessing the cardiovascular relationship with sleep have called into question some of the benefits of treating comorbid sleep disorders, thereby highlighting the need for an ongoing rigorous investigation into how sleep interplays with systemic diseases. Summary Systemic diseases often have sleep manifestations and this report will help the clinician identify key risk factors linking sleep disorders to systemic diseases so as to optimize the overall care of the patient.
\end{abstract}




\section{Introduction}

All Earth's species maintain a solar 24-h cycle of rest and activity, and disrupting the cycle affects adaptation and homeostasis. Sleep's quotidian "normalness" means that analogous to fish not knowing about water until it is dry, sleep is not commonly thought about until it is disrupted.

For example, about $30 \%$ of the adult population complain of transient insomnia, and about $10 \%$ experience chronic insomnia that disrupts daytime function [1]. Patients with chronic insomnia experience less work productivity, more absenteeism, more accidents, and more hospitalizations, leading to direct treatment costs of approximately $\$ 60 \mathrm{~B}$ annually [2]. Considering the potential widespread reach of comorbid sleep disorders, evaluating sleep in the neurological patient is important.

This review will introduce the accepted organization of sleep disorders, review important features in history taking and evaluation, and survey the systemic diseases that have important comorbidities with particular sleep disorders.

\section{General considerations}

\section{Classification of sleep disorders}

An abridged listing of sleep disorders from the American Academy of Sleep Medicine (Table 1) provides an overview of the current classification [3].

Insomnia is a chronic dissatisfaction with sleep duration and quality that is associated with daytime dysfunction. Although pharmacologic treatment is often pursued for chronic insomnia management, outcomes are often better addressing underlying factors with the early use of cognitive-behavioral therapy for insomnia (CBT-i) [1].

Sleep-related breathing disorders involve dysfunction of the respiratory system during sleep, usually resulting in daytime hypersomnia. Obstructive sleep apnea (OSA), central sleep apnea (CSA), and respiratory effort related arousals are classified under this category. Treatment options including continuous positive airway pressure (CPAP), positional therapy, mandibular advancement devices, healthy weight loss, and even a novel cranial nerve stimulator which protrudes the tongue forward during sleep $[4 \bullet \bullet]$.

Central hypersomnias are defined as a primary dysregulation of sleep resulting from dysfunction of the central nervous system that causes daytime hypersomnia. Often treatment addresses the underlying cause and may include use of strategic napping and wake-promoting medications.

Circadian disorders consist of various lesions or external disruptions of the circadian timing system that desynchronize the brain's clock from the external solar light-dark cycle, resulting in hypersomnia or insomnia in a clockdependent fashion. Treatment of circadian rhythm disorders involves adjusting life around the patient's desired sleep time or augmenting factors that entrain the body's clock.

Parasomnias represent disorders of faulty inhibition of waking behaviors that arise inappropriately during sleep and are divided into those that occur during nonREM sleep, REM sleep, or state transitions. REM sleep behavior disorder is a parasomnia characterized by loss of muscle atonia during REM sleep that usually occurs in patients with neurodegenerative disorders. It is often treated effectively addressing other sleep disturbances and treating with clonazepam or melatonin [5]. 
Table 1. Abridged classification of the AASM sleep disorders

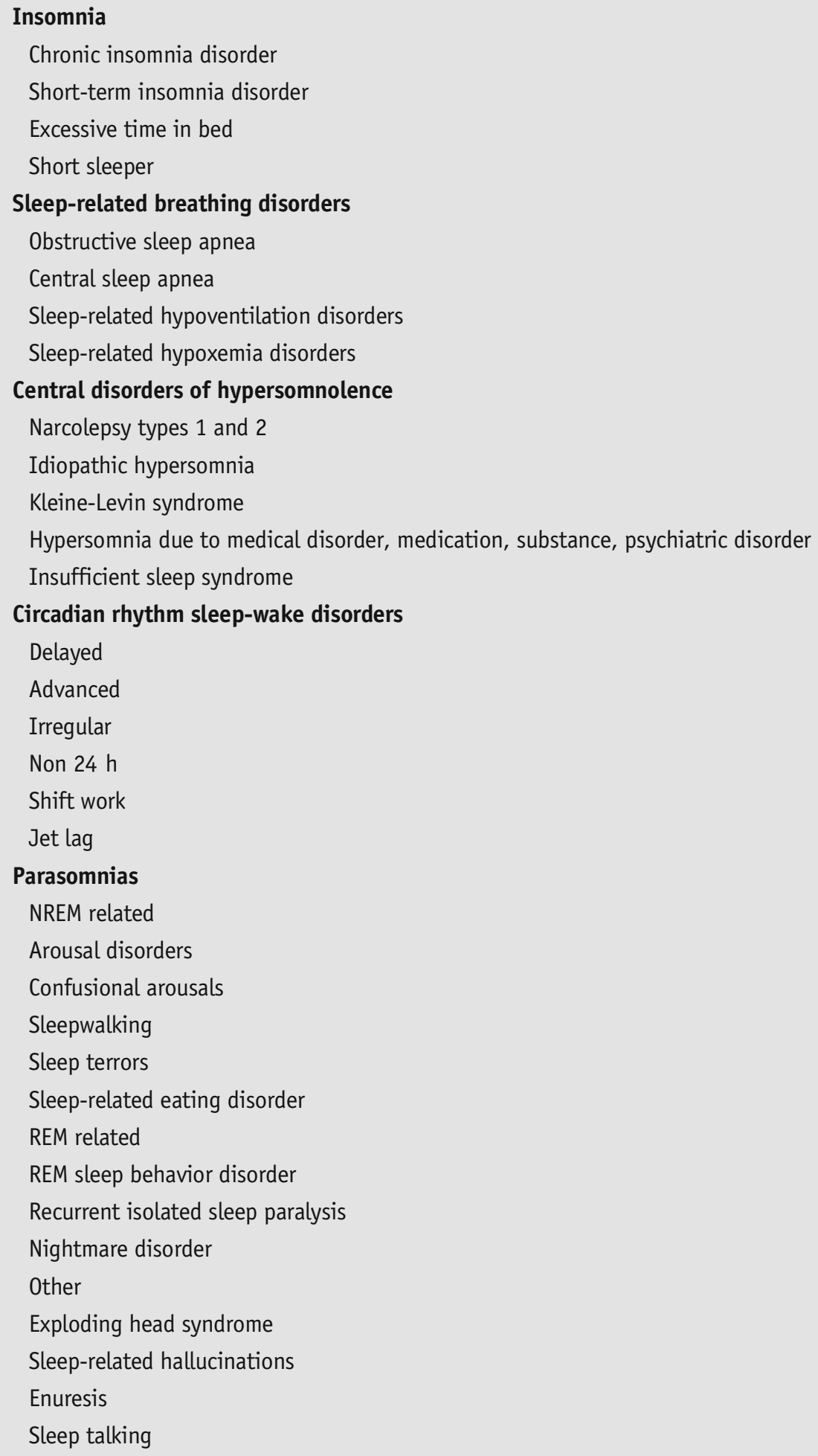


Table 1. (Continued)

\author{
Restless legs syndrome \\ Periodic limb movement disorder \\ Leg cramps \\ Bruxism \\ Rhythmic movement disorder \\ Benign sleep myoclonus of infancy \\ Propriospinal myoclonus at sleep onset \\ Normal variants
}

Sleep-related movement disorders consist of fragmentary, often repetitive body movements that can disrupt sleep or, sometimes worse, disturb the sleep of bed partners. Periodic limb movement disorder (PLMD) and restless legs syndrome (RLS) both fall under this category and are treated with repletion of iron stores and consideration of dopaminergic agonists [6].

\title{
Sleep history
}

A sleep history helps a patient disclose sleep findings and helps the physician organize it into categories of hypersomnia, sleep habits and scheduling, sleep characteristics, environmental issues, and sleep interrupters (Table 2).

The Epworth Sleepiness Scale quantifies the degree of hypersomnia [7]. Most adults require 7-9 h of daily sleep [8] and prefer it organized into either a monophasic, nocturnal schedule or in a biphasic pattern augmented with an afternoon "siesta." The sleep pattern characterizes the presence and severity of sleep-onset insomnia, sleep maintenance insomnia, or terminal insomnia (insomnia distributed within the last half of the sleep period). "Catch-up" sleep, a phenomenon of prolonged sleep on a free day, is a classic sign of sleep deprivation. Habitual early-phase advances ("morning larks"), late-phase delays ("night owls"), or a chaotic, irregular schedule can be a sign of circadian disorders. One also must inquire about common sleep disruptors including leg movements, snoring, witnessed apneas, and environmental factors.

\section{Diagnostic testing modalities}

The sleep diary, often available through standardized forms or even websites or smartphone apps, consists of 1-2 weeks of self-reported sleep times.

The overnight polysomnography (PSG) is the gold-standard measurement of sleep architecture, respiratory disorders such as OSA, and parasomnias. In the case of OSA, the unattended (home) sleep study has had an 


\section{Table 2. A categorical sleep history}

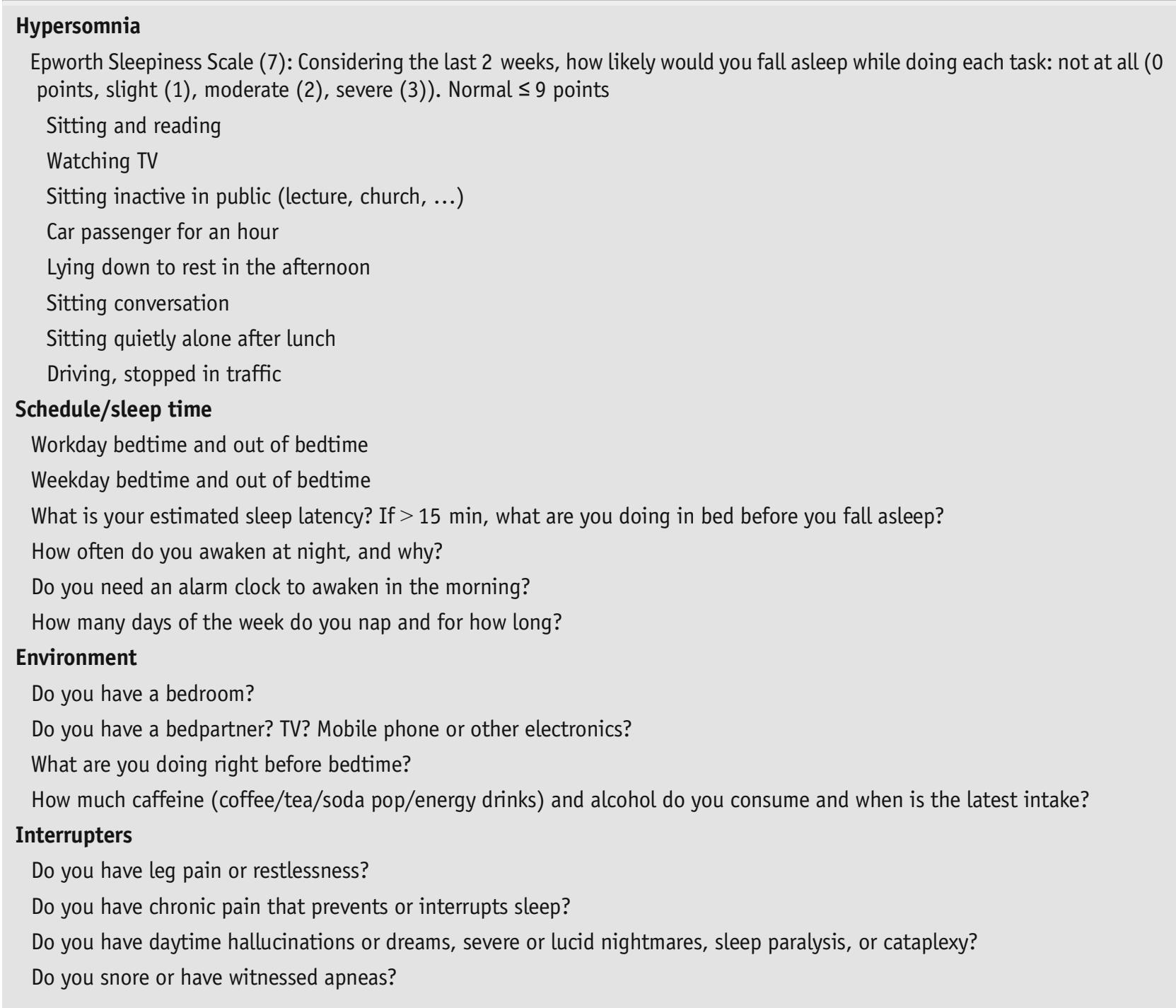

increasing role as a diagnostic testing alternative to the traditional in-lab PSG. Concerns of other sleep disorders (or those that may be present comorbidly with probable OSA) require in-lab PSG that can measure sleep architecture and sleep-associated movements. from which sleep onset is calculated. The test, in combination with PSG performed the night before, is the gold standard in measuring hypersomnia, especially in the evaluation of narcolepsy. 
Wrist actigraphy provides measurements of long-term patterns of rest and activity as proxies for sleep and wakefulness. Such patterns can help to corroborate histories of sleep duration and timing.

Popular smartphones and other ambulatory devices with physiological monitoring capabilities may transform the evaluation of sleep. However, a recent comparison of different brands of activity trackers found that sleepwake measurements varied widely in comparison with sleep diaries or standard PSG [9]. The overall conclusion is that, at the beginning of 2020, wearable devices are not ready for reliable quantification of sleep across individuals. Although serial recordings confined to a single individual may hold some value, these measurements have yet to be validated.

\section{Sleep comorbidities with systemic diseases}

Considering the various sleep disorders and diagnostic tools afforded by a good sleep history and sleep testing, understanding the relationship between sleep disorders and systemic diseases has far-reaching implications in optimizing the care of the patient. The following sections will address sleep manifestations of various neurological disorders arising from systemic disease based on organ system.

Endocrine disorders

\section{Thyroid disease}

Almost half of the patients with hypothyroidism report at least one sleep complaint such as restless sleep, choking, hypersomnia, or fatigue [10]. OSA is present in approximately $30 \%$ [11]. A unique mechanism of airway restriction in hypothyroidism is myxedematous mucoprotein deposition in the airway's soft tissues and dilator muscles even though myxedema can be absent [12]. Larger goiters can also cause OSA by external compression of the airway [13].

On the other side of the thyroid spectrum, hyperthyroidism is most closely associated with insomnia, occurring in $37 \%$ of patients [14]. Arousal disorders-specifically, sleep walking-also occur, especially in the setting of thyrotoxicosis [15], proposed to arise from frequent arousals and impairment of attaining slow-wave sleep as the direct result of thyroid hormone.

Beyond the treatment of the specific sleep disorder, sleep problems usually remit following appropriate treatment of the underlying thyroid disorder [15].

\section{Type 2 diabetes mellitus}

Sleep disorders affect high proportions of those with type 2 diabetes mellitus (DM): surveys of patients with DM compared with those of controls show a 
nearly 2-fold propensity for insomnia, fourfold higher use of sedative-hypnotics, and a 10-fold higher rate of hypersomnolence [16]. OSA is highly prevalent in $\mathrm{DM}$, and many are undiagnosed [17]. Contributors to a multifactorial series of sleep disruptors include periodic limb movements and restless legs syndrome (RLS), diabetic neuropathy, and fluctuations in blood glucose [18].

DM presents an excellent model by which to demonstrate the reciprocal effects of sleep disruption on the primary disease. First, sleep disturbances affect the regulation of the neuroendocrine control of appetite. Sleep deprivation promotes overeating through hyperactivity of orexin system [19] and activates the hypothalamic-pituitary-adrenal system to increase cortisol secretion resulting in impaired glucose tolerance [20,21]. These multiple mechanisms support clinical observations that untreated OSA may be reason for the ineffective treatment of DM, and that accordingly, treatment with CPAP leads to improvements in glycemic control in some patients [22].

\section{Sex hormones}

Sex hormones and gender affect the distribution and susceptibility to a variety of sleep disorders. Men, on the basis of relative airway collapsibility, have approximately a twofold increased risk of OSA compared with women (15$30 \%$ in males and $10-15 \%$ in females) [23]. A potential side effect in the treatment of hypoandrogenism is the facilitation of OSA given the impact testosterone has on upper airway collapsibility [24].

Testosterone levels may affect the propensity for chronic insomnia. Men with hypoandrogenism demonstrate reduced sleep efficiency, increased nighttime awakenings, and reduced deep sleep compared with the normaltestosterone-level controls, although it is not clear whether these features improve with testosterone therapy [25]. Women experience higher rates of chronic insomnia (risk ratio of 1.41 for women versus men) which becomes even more pronounced in the elderly [26]. Despite sleeping longer, overall sleep quality is often lower in women than men [27].

The distribution of sleep disorders in women varies with reproductive lifespan. Younger women are more susceptible to restless legs syndrome (RLS), mainly on the basis of menses-associated iron-deficiency. During pregnancy, women are at significantly increased risk for the development of RLS with an overall prevalence exceeding $25 \%$ of all pregnant patients [28]. Treatment of RLS in pregnancy involves iron supplementation with a goal ferritin level $>50 \mathrm{mcg} / \mathrm{l}$. Often, oral iron repletion is adequate although there are reports of intravenous iron therapy in severe cases of pregnancy-related RLS and iron-deficiency [29]. Pregnancy is also associated with an increased prevalence of OSA (up to 19\% of pregnant patients during the third trimester) which is associated with increased risks of complications including gestational hypertension, gestational DM, and pre-eclampsia [30]. on sleep form an important aspect of neurological sleep medicine, since many medications that are used by neurologists may affect sleep. Table 3 shows common medications that provoke insomnia, hypersomnolence, respiratory suppression, parasomnias, and RLS/periodic limb movement disorder. 
Table 3. Medication classes (and specific examples) that can cause sleep disturbances

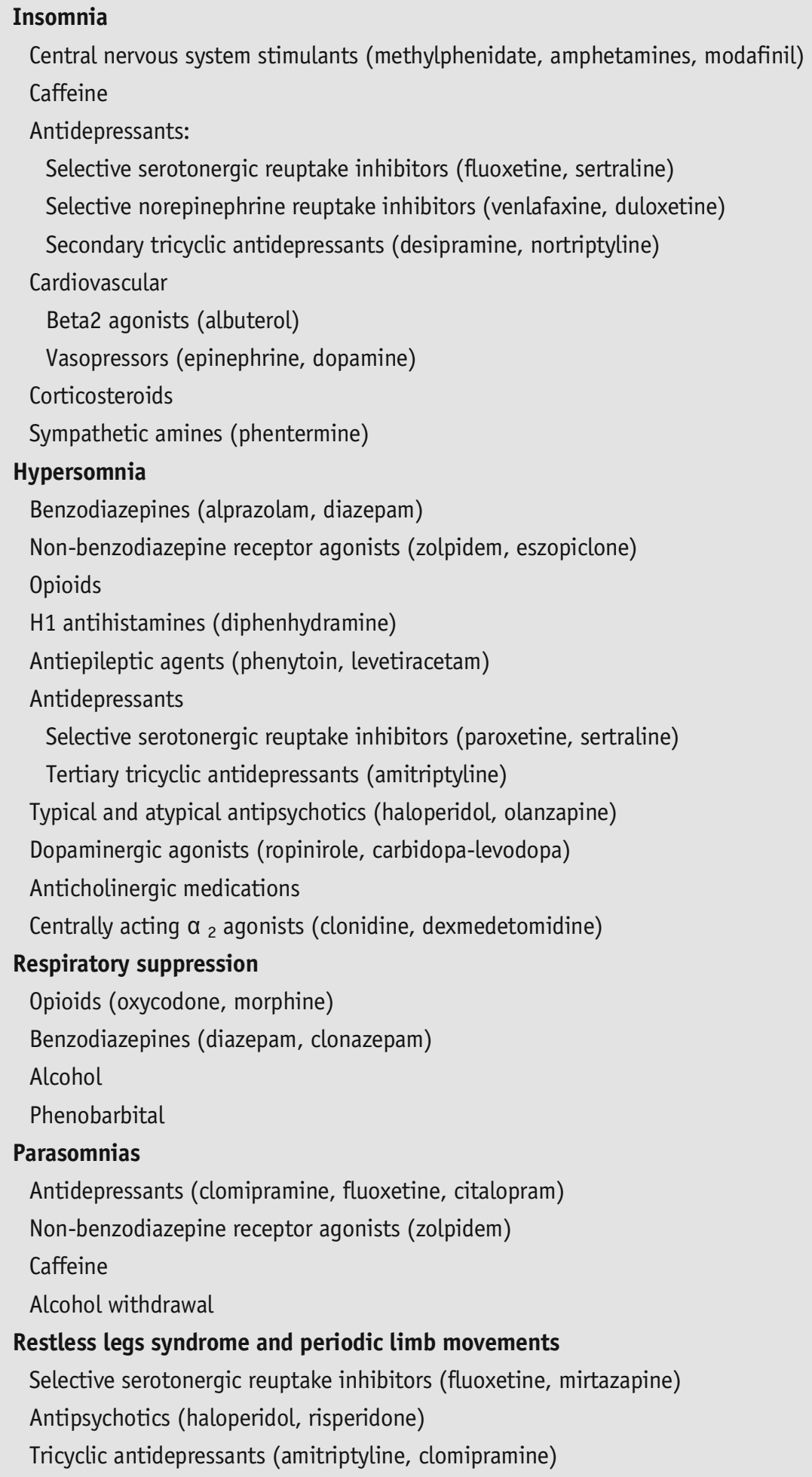

\section{Parasomnias}

Antidepressants (clomipramine, fluoxetine, citalopram)

Non-benzodiazepine receptor agonists (zolpidem)

Caffeine

Alcohol withdrawal

\section{Restless legs syndrome and periodic limb movements}

Selective serotonergic reuptake inhibitors (fluoxetine, mirtazapine)

Antipsychotics (haloperidol, risperidone)

Tricyclic antidepressants (amitriptyline, clomipramine) 
Sleep disturbances are highly prevalent in patients with chronic kidney disease (CKD) spanning the broad spectrum of sleep disorders including hypersomnia, insomnia, sleep-related breathing, and RLS.

The prevalence of OSA in CKD ranges from 25 to $70 \%$, rates that are not explained solely by overlapping comorbidities common to both OSA and CKD [31]. The co-occurrence of both CKD and OSA is associated with increased cardiovascular events and all-cause mortality [32-34]. Usually, OSA develops in patients with CKD independent of underlying renal dysfunction, but some evidence shows that CKD can cause or exacerbate OSA and central sleep apnea. Proposed mechanisms for this causal relationship include uremic neuropathy, altered chemosensitivity, and hypervolemia [35]. Accordingly, renal replacement therapy and fluid removal [36] may improve obstructive or central sleep apnea. Conversely, treatment of sleep apnea with PAP may improve renal function in those with borderline renal impairment [37].

RLS is a common and debilitating symptom in patients with CKD, occurring in up to $25 \%$ of patients on hemodialysis compared with that in approximately $7 \%$ of the general population [38]. Although RLS symptoms generally follow a circadian rhythmicity with increased symptoms occurring at night, RLS symptoms can occur during the long periods of daytime inactivity during hemodialysis [39]. Treatment is primarily focused on ensuring adequate iron stores then considering medical therapy, as per routine care of RLS.

Sleep disorders and infectious diseases have few specific associations. In general, acute infection is associated with mild encephalopathy that masquerades as hypersomnolence and fatigue. Pro-inflammatory cytokines are implicated in the development of these constitutional symptoms. Some infections, however, directly affect regulatory centers of the sleep-wake system.

Encephalitis lethargica is a historical, pandemic cause of hypersomnolence of renewed interest since this review is being written in the middle of the COVID19 pandemic. Also known as Von Economo's encephalitis, it occurred in association with the Spanish flu pandemic of 1918 [40]. An estimated 1 million were affected worldwide. The most common subtype, the somnolentophthalmoplegic form, developed after flu-like symptoms of fever and malaise and consisted of subsequent ophthalmoplegia accompanied by long periods of hypersomnia. Despite the appearance of deep sleep, patients could be easily awoken and sometimes maintained memories of activities that had transpired around them while "asleep." This state of acute akinetic psuedosomnulence could be followed by the development of chronic postencephalitic parkinsonism.

The pandemic associated with the severe acute respiratory syndrome coronavirus 2 (SARS-CoV-2; i.e., COVID-19), occurring during the writing of this review, features evolving literature. The first reports centered on respiratory symptoms. Although the involvement of the nervous system now appears prevalent [41], sleep disorders have yet to be specifically reported. However, the psychological responses to social distancing, change in schedules, and other features of an active pandemic have caused a wave of anxiety and depression, which in turn have been associated with poor sleep quality. For example, a 
survey of 1250 Chinese health care workers showed prevalences of depression at 50\%, anxiety at 45\%, and insomnia at 34\% [42].

Postinfectious or postvaccination narcolepsy is rare but is important in developing overall hypotheses in the etiology of idiopathic narcolepsy. In 2009, certain vaccinations in Europe for the H1N1 pandemic caused narcolepsy at a risk of $1: 16,000$ in pediatric patients [43]. Fortunately, the risk of postvaccination narcolepsy appeared confined to specific vaccine formulations. The incident, however, has led to ongoing research in the immunological etiology of narcolepsy.

African trypanosomiasis, or sleeping sickness, remains important in the developing world. It is a parasitic infection spread by the tsetse fly that is endemic in sub-Saharan Africa. The first symptoms include fever, headaches, and lymphadenopathy. Once the parasite enters the central nervous system, disordered, fragmented sleep ensues often with inversion of the circadian sleep-wake cycle. The World Health Organization outlines treatment with a regimen of antiparasitic medications once symptoms have started [44].

Non-alcoholic fatty liver disease (NAFLD) consists of idiopathic hepatic steatosis with a prevalence of 17 to $33 \%$ of the general population with increased frequency in individuals with obesity or DM [45]. Given these co-associations, OSA is common. Untreated OSA may exacerbate liver injury because of oxidative stress and systemic inflammation [46] and is a risk in conversion from NAFLD to liver fibrosis [47]. Trials with CPAP have shown inconsistent results in markers of liver injury following treatment of OSA [48].

The symptoms of gastroesophageal reflux disease (GERD) worsen during sleep, particularly if sleep occurs soon after a meal [49]. The lower esophageal sphincter that normally prevents reflux may be compromised by the increase in thoracic pressure in the setting of the upper airway obstruction [49]. Patients with symptoms of GERD should be screened for OSA, and conversely, interruption of sleep in absence of OSA may improve with treatment with a proton pump inhibitor (PPI) [50] or by simply elevating the head of the bed.

Inflammatory bowel disease (IBD) has bilateral interactions with sleep [51]. Given the relationship between sleep deprivation/fragmentation on cytokine regulation and immune dysfunction, it is hypothesized that poor sleep quality worsens overall symptoms of IBD $[52,53]$. Additionally, the pro-inflammatory state disrupts the circadian rhythm [54]. Subjective and objective measurements of sleep quality and timing should be considered in patients with IBD particularly in those who have frequent inflammatory flares despite otherwise adequate management. An algorithmic approach to sleep assessment in IBD patients has been proposed by Canakis et al. [51].

Systemic lupus erythematosus and rheumatoid arthritis serve as the prototypical diseases of this group of disorders, with a prevalence of sleep disturbances of greater than 50\% [55]. The mechanisms of sleep disturbances, as well as the reciprocal relationship in the contribution of poor sleep to worse autoimmune status, are thought to be similar to those described above with IBD $[56,57]$. The specific sleep disorders prevalent in this group are OSA and periodic limb 
movement disorder (PLMD) (both with greater than $25 \%$ prevalence) $[58,59]$. As seen above, hypersomnolence and activity-limiting fatigue arise from specific sleep disorders, pain, and medication side effects well as the primary effects of the primary pro-inflammatory status [60,61]. Often treating the underlying autoimmune disorder improves associated fatigue. However, if sleepiness persists, then evaluating for a comorbid sleep disorder such as obstructive sleep apnea is indicated.

One syndrome with possible autoimmune origins is chronic fatigue syndrome. Sleep disturbances, insomnia, and unrefreshing sleep are common symptoms, yet patients rarely report relief despite appropriate identification and treatment of comorbid sleep disorders [62]. Cognitive-behavioral therapy (CBT) and graded exercise therapy are commonly pursued treatment approaches [62].

Obstructive lung diseases (most commonly asthma, chronic obstructive pulmonary disease (COPD), and less common disorders such as cystic fibrosis (CF) or bronchiolitis obliterans) may affect nocturnal ventilation. OSA and COPD often overlap given shared body habitus and other mutual risk factors; estimates of comorbid OSA and COPD range from 7 to 66\% [63]. Patients with severe COPD treated with nocturnal noninvasive ventilation (NIPPV; a more advanced form of positive airway pressure) experience an absolute risk reduction of $17 \%$ of the risk of hospital readmission or death at 12 months, compared with those treated with standard care and without NIPPV [64•].

Insomnia is another common complaint among patients with COPD. Circadian bronchial constriction may cause nocturnal wheezing, dyspnea, or other symptoms of asthma prompting the patient to awaken [65]. In addition, the hyperadrenergic response to beta 2 agonist inhalers used in treatment for acute dyspnea impairs sleep onset (see Table 3).

The growing success in treatments for CF patients means that sleep disorders arising from their intrinsic obstructive lung disease are now coming to the attention of caregivers. Many factors contribute to sleep disruption including chronic cough, frequent infections, abdominal discomfort, reflux, frequent stools, medication side effects, and psychological disease [66]. In addition to sleep disruption, patients with CF are susceptible to hypoventilation that worsens with disease progression. Use of NIPPV in high-risk patients with hypercapnia has been shown to improve physiologic parameters and at times can positively impact symptoms, particularly in patients who have severe disease while awaiting lung transplant [67].

Restrictive lung diseases (defined by a reduced total lung capacity) include those with parenchymal damage such as idiopathic fibrosis, hypersensitivity pneumonitis, or other interstitial pneumonias. Alternatively, lung parenchyma is normal in restrictive diseases such as obesity hypoventilation syndrome, hemi-diaphragm paresis, or neuromuscular disorders (muscular dystrophies, amyotrophic lateral sclerosis). Restrictive lung disease patients, as seen above with obstructive disease patients, are susceptible to nocturnal hypoventilation, subsequent $\mathrm{CO}_{2}$ retention, and compensatory sleep fragmentation. Use of NIPPV in patients with severe restrictive lung disease (spanning obesity hypoventilation syndrome to muscular dystrophies and ALS) has had positive impacts on survival and quality of life [68, 69]. 
Over $40 \%$ of patients with congestive heart failure (CHF) have comorbid OSA mainly on the basis of mutual risk factors of DM, hypertension, obesity, and older age $[70,71]$. In addition, insomnia in those with CHF may arise from a variety of factors including diuretic medications (and subsequent nocturia), positional heart failure symptoms, increased adrenergic status, or psychosocial factors [72]. Treatments addressing comorbid OSA and insomnia improve sleep quality but demonstrate mixed results in terms of long-term cardiovascular outcomes $[72,73]$.

Patients with acute myocardial infarction (AMI) experience both acute and chronic sleep disorders. Due to the circadian variability of adrenergic hormones and cardiac and systemic vasculature [74], the timings of AMI, sudden cardiac death, and arrhythmia occur with increased frequency at night [75]. Cardiac ischemia may present a series of nocturnal symptoms including paroxysmal dyspnea, chest pain, agitation, or insomnia. Surviving patients are at risk for chronic sleep disorders such as insomnia and sleep-disordered breathing with or without the co-occurrence of anxiety or depression [76].

Retrospective, longitudinal data demonstrate that those with OSA and who are adherent with CPAP experience improved cardiovascular morbidity and mortality over non-adherent patients [77]. However, these findings have not been clearly supported by prospective, randomized trials. The Sleep Apnea cardioVascular Endpoints Trial (SAVE Trial) has called into question the causal link between the treatment of OSA and cardiovascular outcomes. With a mean follow-up of 3.7 years, those randomized to PAP experienced no significant improvements in study endpoints of death from cardiovascular causes, AMI, stroke, and hospitalization for unstable angina, CHF, or transient ischemic

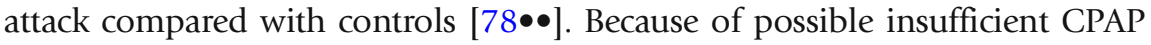
use and because of the lack of main indications for CPAP treatment (such as severe sleepiness), interpretation of the findings of this large trial remains controversial. In practice, these authors often pursue CPAP treatment for patients with OSA and cardiovascular risk factors (even in the absence of sleepiness) at least for a trial period to assess adherence to treatment and to determine if there are subjective and objective improvements to sleep quality.

With a prevalence range of $21-74 \%$, OSA is common in patients with atrial fibrillation and other arrhythmias [79]. Accordingly, the Sleep Heart Health Study showed a two- to fivefold higher risk of arrhythmia in patients with severe OSA compared with that in controls [80]. Retrospective series show that, in patients with atrial fibrillation and untreated OSA, the risk of atrial fibrillation recurrence following cardioversion is $82 \%$ compared with $42 \%$ in patients who are adherent to CPAP [81]. However, a prospective randomized control trial called retrospective findings into question [82]. Similar in design to the SAVE Trial, patients with atrial fibrillation were randomized to CPAP versus usual therapy from a cohort in which sleepiness was specifically excluded. This small trial (25 total) assessed the primary outcome of time to arrhythmia recurrence. Both arms had recurrence rates of $25 \%$. Although the trial showed that CPAP itself provides no specific benefit to those with atrial fibrillation, the outcomes for treatment of those with both disorders remain unclear.

Although the above studies centered on associations between cardiac disease and OSA, patients with CHF, AMI, and atrial fibrillation experience high rates of 
central sleep apnea (CSA) as well, exceeding 35\% in patients with mild symptomatic CHF as an example [83]. Cheyne-Stokes respiration, a cyclical form of CSA, results when circulatory impairment perturbs the normal responsiveness in respiratory control resulting in alterations in "the loop gain" in modulating changes in carbon dioxide and oxygen levels in the bloodstream [84], analogous to overly aggressive adjustments to a thermostat in response to changing temperature. The presence of CSA has been considered a marker of increased mortality in patients with CHF, although aims to resolve the treatment of CSA (with CPAP or more advanced modalities) have not clearly demonstrated an improvement in cardiovascular outcomes [85].

Estimates of the prevalence of sleep disturbances across cancer patients range widely from 25 to $95 \%[86,87]$. Insomnia is the most common disorder with prevalence levels ranging from 30 to $50 \%$ [86, 88]. Patients with cancer who undergo PSG have shorter total sleep times, longer times in bed, low sleep efficiency, and proportionately less deep sleep than controls [88]. Insomnia in patients with cancer is driven by a multitude of factors including pre-existing socioeconomic and psychiatric disorders, fatigue, age, RLS, pain, and medication effects [86, 89]. Treatment follows that for the general population. Although sedative-hypnotics are most commonly prescribed, no evidence exists for specific pharmacologic interventions for sleep disturbances in this population [90]. Cognitive-behavioral therapy is currently the recommended first-line treatment for chronic insomnia [91]. Because the rarity of trained psychologists makes finding a provider difficult in some circumstances, the electronic delivery of cognitive-behavioral therapy has been sought as an alternative to face-to-face therapy $[92,93]$.

The bilateral interactions between sleep and critical illness form a rapidly changing area of investigation which is made particularly challenging given the difficulties in measuring sleep in critically ill patients $[94,95]$. Lack of sleep-or its encephalopathic analog-may affect outcomes in critical illnesses. For example, a lack of scorable REM sleep correlates with longer ventilator weaning time compared with controls with intact REM [96]. Failure rates on noninvasive ventilation are impacted by sleep continuity [97]. Delirium, a common neurobehavioral syndrome seen in upwards of $80 \%$ of patients in the ICU $[98,99]$, is associated with significantly worse outcomes in the ICU including longer hospital stay and increased mortality [100]. In turn, disrupted circadian rhythms are a strong risk factor for the development of delirium [101].

Ongoing work may establish that the optimization of circadian rhythmicity can directly improve ICU outcomes. The most widespread technique is to provide hospital environments that enable entrainment to a stable circadian rhythm. Exposure to daylight, mitigation of noise, stimulation, and lights during "quiet periods" have been established as good practice in many ICUs [102].

Other investigations have centered on pharmacological interventions. Melatonin, a hormone with important regulatory interactions with the main clock, has been employed to optimize sleep and circadian rhythm in the ICU. However, a small randomized trial of 3 nights therapy with melatonin versus placebo did not alter behavioral sleep or the need for sedation for delirium [103]. 
Perhaps more promising is the use of dexmedetomidine, a potent selective alpha-2 adrenergic receptor agonist, which provides what appears to be adequate sedation without obliteration of underlying sleep-wake cycles. A prospective study of patients on mechanical ventilation with delirium was randomized to receive dexmedetomidine or placebo in addition to standard medical care [104]. Patients in the treatment arm had a significant increase in the number of ventilator-free hours at 7 days and shorter duration of delirium when compared with the placebo. Although this trial suggests a role for dexmedetomidine in the treatment of delirium in mechanically ventilated ICU patients, those in the treatment arm also required reduced amounts of other sedatives, and therefore, its benefit may be secondary rather than primary. Nevertheless, dexmedetomidine may serve as a less "deliriogenic" sedative agent in the ICU.

Some disorders specifically disrupt the thalamic regulation of sleep, present with primary sleep disturbances, and have a variety of systemic or hereditary causes and thus are difficult to classify in our system-based organization used above. Agrypnia excitata is a syndrome of unrelenting insomnia, autonomic hyperactivity, agitated delirium, and oneiric stupor (dream-like movements and hallucinations) [105]. Polysomnography of those with agrypnia excitata will show the absence of slow-wave sleep and sleep spindles with fluctuating, poorly sustained wake, stage N1, and REM sleep stages. Fatal familial insomnia is an autosomal dominant disorder caused by a mutation of the prion protein. Patients experience progressive, subacute dementia, delirium, and insomnia. Treatment is supportive. Morvan's syndrome is a rare autoimmune disorder that is caused by antibodies to voltage-gated potassium channels. Patients present with acute onset of insomnia along with myokymia and diaphoresis that progresses at variable rates and usually is fatal without treatment. Most have high levels of immunoglobulin and respond to plasmapheresis. Finally, an unfortunately common syndrome is delirium tremens that arises acutely within $48 \mathrm{~h}$ of cessation of alcohol in abusers. The onset of ophthalmoparesis and ataxia denotes Wernicke's encephalopathy. Unless prophylactically treated with thiamine and appropriate inhibitory alcohol analogs (such as benzodiazepines), permanent sequelae in the form of hippocampal degeneration may occur (Korsakov's syndrome: profound and permanent anterograde amnesia).

A sleep history is the first step in the evaluation of a patient with systemic disease who presents with "trouble sleeping." Patients with hypersomnia that is not easily attributable to insufficient sleep should be evaluated with overnight polysomnography, or in certain patients with isolated risk factors for OSA-home sleep testing. The mainstay treatment for OSA is positive airway pressure, especially if underlying systemic disease prevents modification of the underlying risk factors of obesity. Insomnia is best treated with cognitive behavioral therapy that features identification of poor sleep habits, but limited use of sedative-hypnotics can also benefit patients. Sorting out the sleeping environment, whether at home or in the hospital, can improve sleep. 


\section{References and Recommended Reading}

Papers of particular interest, published recently, have been

highlighted as:

- Of importance

- Of major importance

1. Morin CM, LeBlanc M, Daley M, Gregoire JP, Merette C Epidemiology of insomnia: prevalence, self-help treatments, consultations, and determinants of helpseeking behaviors. Sleep Med. 2006;7(2):123-30.

2. Chilcott LA, Shapiro CM. The socioeconomic impact of insomnia. An overview. Pharmacoeconomics. 1996;10 Suppl 1:1-14.

3. Sateia MJ. International classification of sleep disorders-third edition: highlights and modifications. Chest. 2014;146(5):1387-94.

4.• Strollo PJ Jr, Soose RJ, Maurer JT, de Vries N, Cornelius J, Froymovich $\mathrm{O}$, et al. Upper-airway stimulation for obstructive sleep apnea. N Engl J Med. 2014;370(2):139-4.

This trial highlights novel technology which led to FDA-approved treatment of moderate to severe obstructive sleep apnea with a hypoglossal nerve stimulator in patients intolerant to CPAP.

5. Kunz D, Mahlberg R. A two-part, double-blind, placebo-controlled trial of exogenous melatonin in REM sleep behaviour disorder. J Sleep Res. 2010;19(4):591-6.

6. Salminen AV, Winkelmann J. Restless legs syndrome and other movement disorders of sleep-treatment update. Curr Treat Options Neurol. 2018;20(12):55.

7. Johns M. A new method for measuring daytime sleepiness: the Epworth sleepiness scale. Sleep. 1991; 14:540-5.

8. Watson NF, Badr MS, Belenky G, Bliwise DL, Buxton OM, Buysse D, et al. Recommended amount of sleep for a healthy adult: a joint consensus statement of the American Academy of Sleep Medicine and Sleep Research Society. Sleep. 2015;38(6):843-4.

9. Gruwez A, Bruyneel AV, Bruyneel M. The validity of two commercially-available sleep trackers and actigraphy for assessment of sleep parameters in obstructive sleep apnea patients. PLoS One. 2019;14(1):e0210569.

10. Pelttari L, Rauhala E, Polo O, Hyyppa MT, Kronholm E, Viikari J, et al. Upper airway obstruction in hypothyroidism. J Intern Med. 1994;236(2):177-81.

11. Jha A, Sharma SK, Tandon N, Lakshmy R, Kadhiravan $\mathrm{T}$, Handa KK, et al. Thyroxine replacement therapy reverses sleep-disordered breathing in patients with primary hypothyroidism. Sleep Med. 2006;7(1):5561.

12. Attal P, Chanson P. Endocrine aspects of obstructive sleep apnea. J Clin Endocrinol Metab. 2010;95(2):483-95.
13. Deegan PC, McNamara VM, Morgan WE. Goitre: a cause of obstructive sleep apnoea in euthyroid patients. Eur Respir J. 1997;10(2):500-2.

14. Demet MM, Ozmen B, Deveci A, Boyvada S, Adiguzel $\mathrm{H}$, Aydemir O. Depression and anxiety in hyperthyroidism. Arch Med Res. 2002;33(6):552-6.

15. Giuliano L, Fatuzzo D, Mainieri G, La Vignera S, Sofia $\mathrm{V}$, Zappia M. Adult-onset sleepwalking secondary to hyperthyroidism: polygraphic evidence. J Clin Sleep Med. 2018;14(2):285-7.

16. Skomro RP, Ludwig S, Salamon E, Kryger MH. Sleep complaints and restless legs syndrome in adult type 2 diabetics. Sleep Med. 2001;2(5):417-22.

17. West SD, Nicoll DJ, Stradling JR. Prevalence of obstructive sleep apnoea in men with type 2 diabetes. Thorax. 2006;61(11):945-50.

18. Resnick HE, Redline S, Shahar E, Gilpin A, Newman A, Walter R, et al. Diabetes and sleep disturbances: findings from the Sleep Heart Health Study. Diabetes Care. 2003;26(3):702-9.

19. Taheri S, Lin L, Austin D, Young T, Mignot E. Short sleep duration is associated with reduced leptin, elevated ghrelin, and increased body mass index. PLoS Med. 2004;1:e62.

20. VanHelder T, Symons JD, Radomski MW. Effects of sleep deprivation and exercise on glucose tolerance. Aviat Space Environ Med. 1993;64(6):487-92.

21. Leproult R, Copinschi G, Buxton O, Van Cauter E. Sleep loss results in an elevation of cortisol levels the next evening. Sleep. 1997;20(10):865-70.

22. Aronsohn RS, Whitmore H, Van Cauter E, Tasali E. Impact of untreated obstructive sleep apnea on glucose control in type 2 diabetes. Am J Respir Crit Care Med. 2010;181(5):507-13.

23. Young T, Palta M, Dempsey J, Peppard PE, Nieto FJ, Hla KM. Burden of sleep apnea: rationale, design, and major findings of the Wisconsin Sleep Cohort study. WMJ. 2009;108(5):246-9.

24. Cistulli PA, Grunstein RR, Sullivan CE. Effect of testosterone administration on upper airway collapsibility during sleep. Am J Respir Crit Care Med. 1994;149(2 Pt 1):530-2.

25. Barrett-Connor E, Dam TT, Stone K, Harrison SL, Redline S, Orwoll E, et al. The association of testosterone levels with overall sleep quality, sleep architecture, and sleep-disordered breathing. J Clin Endocrinol Metab. 2008;93(7):2602-9.

26. Zhang B, Wing YK. Sex differences in insomnia: a metaanalysis. Sleep. 2006;29(1):85-93. 
27. Mong JA, Cusmano DM. Sex differences in sleep: impact of biological sex and sex steroids. Philos Trans R Soc Lond Ser B Biol Sci. 2016;371(1688):20150110.

28. Manconi M, Govoni V, De Vito A, Economou NT, Cesnik E, Mollica G, et al. Pregnancy as a risk factor for restless legs syndrome. Sleep Med. 2004;5(3):305-8.

29. Vadasz D, Ries V, Oertel WH. Intravenous iron sucrose for restless legs syndrome in pregnant women with low serum ferritin. Sleep Med. 2013;14(11):1214-6.

30. Liu L, Su G, Wang S, Zhu B. The prevalence of obstructive sleep apnea and its association with pregnancy-related health outcomes: a systematic review and meta-analysis. Sleep Breath. 2019;23(2):399-412.

31. Lin $\mathrm{CH}$, Lurie RC, Lyons OD. Sleep apnea and chronic kidney disease: a state-of-the-art review. Chest. 2020;157(3):673-85.

32. Bello AK, Alrukhaimi M, Ashuntantang GE, Basnet $\mathrm{S}$, Rotter RC, Douthat WG, et al. Complications of chronic kidney disease: current state, knowledge gaps, and strategy for action. Kidney Int Suppl (2011). 2017;7(2):122-9.

33. Xu J, Yoon IY, Chin HJ. The effect of sleep apnea on allcause mortality in nondialyzed chronic kidney disease patients. Sleep Med. 2016;27-28:32-8.

34. Tang SC, Lam B, Yao TJ, Leung WS, Chu CM, Ho YW, et al. Sleep apnea is a novel risk predictor of cardiovascular morbidity and death in patients receiving peritoneal dialysis. Kidney Int. 2010;77(11):1031-8.

35. Abuyassin B, Sharma K, Ayas NT, Laher I. Obstructive sleep apnea and kidney disease: a potential bidirectional relationship? J Clin Sleep Med. 2015;11(8):915-24.

36. Hanly PJ, Pierratos A. Improvement of sleep apnea in patients with chronic renal failure who undergo nocturnal hemodialysis. $\mathrm{N}$ Engl J Med. 2001;344(2):102-7.

37. Puckrin R, Iqbal S, Zidulka A, Vasilevsky M, Barre P. Renoprotective effects of continuous positive airway pressure in chronic kidney disease patients with sleep apnea. Int Urol Nephrol. 2015;47(11):1839-45.

38. Araujo SM, de Bruin VM, Nepomuceno LA, Maximo ML, Daher Ede F, Correia Ferrer DP, et al. Restless legs syndrome in end-stage renal disease: clinical characteristics and associated comorbidities. Sleep Med. 2010;11(8):785-90.

39. Winkelman JW, Chertow GM, Lazarus JM. Restless legs syndrome in end-stage renal disease. Am J Kidney Dis. 1996;28(3):372-8.

40. Hoffman LA, Vilensky JA. Encephalitis lethargica: 100 years after the epidemic. Brain. 2017;140(8):2246-51.

41. Whittaker A, Anson M, Harky A. Neurological manifestations of COVID-19: a systematic review and current update. Acta Neurol Scand. 2020;142(1):14-22.

42. Lai J, Ma S, Wang Y, Cai Z, Hu J, Wei N, et al. Factors associated with mental health outcomes among health care workers exposed to coronavirus disease 2019. JAMA Netw Open. 2020;3(3):e203976.
43. Nohynek H, Jokinen J, Partinen M, Vaarala O, Kirjavainen T, Sundman J, et al. AS03 adjuvanted $\mathrm{AH} 1 \mathrm{~N} 1$ vaccine associated with an abrupt increase in the incidence of childhood narcolepsy in Finland. PLoS One. 2012;7(3):e33536.

44. Organization WH. Trypanosomiasis, human African (sleeping sickness) 2020 [April 14, 2020]. Available from: https:/www.who.int/news-room/fact-sheets/ detail/trypanosomiasis-human-african-(sleepingsickness).

45. Federico A, Dallio M, Masarone M, Persico M, Loguercio C. The epidemiology of non-alcoholic fatty liver disease and its connection with cardiovascular disease: role of endothelial dysfunction. Eur Rev Med Pharmacol Sci. 2016;20(22):4731-41.

46. Lin QC, Chen LD, Chen GP, Zhao JM, Chen X, Huang $J F$, et al. Association between nocturnal hypoxia and liver injury in the setting of nonalcoholic fatty liver disease. Sleep Breath. 2015;19(1):273-80.

47. Jin S, Jiang S, Hu A. Association between obstructive sleep apnea and non-alcoholic fatty liver disease: a systematic review and meta-analysis. Sleep Breath. 2018;22(3):841-51.

48. Rosato V, Masarone M, Dallio M, Federico A, Aglitti A, Persico M. NAFLD and extra-hepatic comorbidities: current evidence on a multi-organ metabolic syndrome. Int J Environ Res Public Health. 2019;16(18).

49. Lim KG, Morgenthaler TI, Katzka DA. Sleep and nocturnal gastroesophageal reflux: an update. Chest. 2018; 154(4):963-71.

50. Orr WC, Goodrich S, Robert J. The effect of acid suppression on sleep patterns and sleep-related gastro-oesophageal reflux. Aliment Pharmacol Ther. 2005;21(2):103-8.

51. Canakis A, Qazi T. Sleep and fatigue in IBD: an unrecognized but important extra-intestinal manifestation. Curr Gastroenterol Rep. 2020;22(2):8.

52. Gombert M, Carrasco-Luna J, Pin-Arboledas G, Codoner-Franch $\mathrm{P}$. The connection of circadian rhythm to inflammatory bowel disease. Transl Res. 2019;206:107-18.

53. Krueger JM, Majde JA, Rector DM. Cytokines in immune function and sleep regulation. Handb Clin Neurol. 2011;98:229-40.

54. Liu X, Yu R, Zhu L, Hou X, Zou K. Bidirectional regulation of circadian disturbance and inflammation in inflammatory bowel disease. Inflamm Bowel Dis. 2017;23(10):1741-51.

55. Palagini L, Tani C, Mauri M, Carli L, Vagnani S, Bombardieri S, et al. Sleep disorders and systemic lupus erythematosus. Lupus. 2014;23(2):115-23.

56. Irwin MR, Olmstead R, Carrillo C, Sadeghi N, Fitzgerald JD, Ranganath VK, et al. Sleep loss exacerbates fatigue, depression, and pain in rheumatoid arthritis. Sleep. 2012;35(4):537-43.

57. Irwin MR, Wang M, Campomayor CO, ColladoHidalgo A, Cole S. Sleep deprivation and activation of morning levels of cellular and genomic markers of 
inflammation. Arch Intern Med. 2006;166(16):175662.

58. Valencia-Flores M, Resendiz M, Castano VA, Santiago V, Campos RM, Sandino S, et al. Objective and subjective sleep disturbances in patients with systemic lupus erythematosus. Arthritis Rheum.

1999;42(10):2189-93.

59. Vina ER, Green SL, Trivedi T, Kwoh CK, Utset TO.

Correlates of sleep abnormalities in systemic lupus: a cross-sectional survey in an urban, academic center. J Clin Rheumatol. 2013;19(1):7-13.

60. Tench CM, McCurdie I, White PD, D'Cruz DP. The prevalence and associations of fatigue in systemic lupus erythematosus. Rheumatology (Oxford). 2000;39(11):1249-54.

61. Da Costa D, Bernatsky S, Dritsa M, Clarke AE, Dasgupta K, Keshani A, et al. Determinants of sleep quality in women with systemic lupus erythematosus Arthritis Rheum. 2005;53(2):272-8.

62. Russell C, Kyle SD, Wearden AJ. Do evidence based interventions for chronic fatigue syndrome improve sleep? A systematic review and narrative synthesis. Sleep Med Rev. 2017;33:101-10.

63. Shawon MS, Perret JL, Senaratna CV, Lodge C, Hamilton GS, Dharmage SC. Current evidence on prevalence and clinical outcomes of co-morbid obstructive sleep apnea and chronic obstructive pulmonary disease: a systematic review. Sleep Med Rev. 2017;32:58-68.

64. Murphy PB, Rehal S, Arbane G, Bourke S, Calverley PMA, Crook AM, et al. Effect of home noninvasive ventilation with oxygen therapy vs oxygen therapy alone on hospital readmission or death after an acute COPD exacerbation: a randomized clinical trial. JAMA 2017;317(21):2177-86

Prospective trial highlighting positive impact of noninvasive ventilation on hospital readmission rates or death in patients with severe chronic obstructive pulmonary disease.

65. Martin RJ. Nocturnal asthma: circadian rhythms and therapeutic interventions. Am Rev Respir Dis. 1993;147(6 Pt 2):S25-8.

66. Bouka A, Tiede H, Liebich L, Dumitrascu R, Hecker C, Reichenberger F, et al. Quality of life in clinically stable adult cystic fibrosis out-patients: associations with daytime sleepiness and sleep quality. Respir Med. 2012;106(9):1244-9.

67. Young AC, Wilson JW, Kotsimbos TC, Naughton MT. Randomised placebo controlled trial of non-invasive ventilation for hypercapnia in cystic fibrosis. Thorax. 2008;63(1):72-7.

68. Priou P, Hamel JF, Person C, Meslier N, Racineux JL, Urban $\mathrm{T}$, et al. Long-term outcome of noninvasive positive pressure ventilation for obesity hypoventilation syndrome. Chest. 2010;138(1):8490

69. Bourke SC, Tomlinson M, Williams TL, Bullock RE, Shaw PJ, Gibson GJ. Effects of non-invasive ventilation on survival and quality of life in patients with amyotrophic lateral sclerosis: a randomised controlled trial. Lancet Neurol. 2006;5(2):140-7.
70. Collen J, Lettieri C, Wickwire E, Holley A. Obstructive sleep apnea and cardiovascular disease, a story of confounders! Sleep Breath. 2020.

71. Naughton MT, Bradley TD. Sleep apnea in congestive heart failure. Clin Chest Med. 1998;19(1):99-113.

72. Hayes D Jr, Anstead MI, Ho J, Phillips BA. Insomnia and chronic heart failure. Heart Fail Rev. 2009;14(3):171-82.

73. Mehra R. Sleep apnea and the heart. Cleve Clin J Med. 2019;86(9 Suppl 1):10-8.

74. Crnko S, Du Pre BC, Sluijter JPG, Van Laake LW. Circadian rhythms and the molecular clock in cardiovascular biology and disease. Nat Rev Cardiol. 2019;16(7):437-47.

75. Muller JE, Stone PH, Turi ZG, Rutherford JD, Czeisler $\mathrm{CA}$, Parker $\mathrm{C}$, et al. Circadian variation in the frequency of onset of acute myocardial infarction. N Engl J Med. 1985;313(21):1315-22.

76. Le Grande MR, Jackson AC, Murphy BM, Thomason N. Relationship between sleep disturbance, depression and anxiety in the 12 months following a cardiac event. Psychol Health Med. 2016;21(1):52-9.

77. Marin JM, Carrizo SJ, Vicente E, Agusti AG. Long-term cardiovascular outcomes in men with obstructive sleep apnoea-hypopnoea with or without treatment with continuous positive airway pressure: an observational study. Lancet. 2005;365(9464):1046-53.

78.• McEvoy RD, Antic NA, Heeley E, Luo Y, Ou Q, Zhang X, et al. CPAP for prevention of cardiovascular events in obstructive sleep apnea. N Engl J Med. 2016;375(10):919-31

A randomized clinical trial indicating lack of benefit of using continuous positive airway pressure (CPAP) in high-risk patients on cardiovascular outcomes.

79. Linz D, McEvoy RD, Cowie MR, Somers VK, Nattel S, Levy $\mathrm{P}$, et al. Associations of obstructive sleep apnea with atrial fibrillation and continuous positive airway pressure treatment: a review. JAMA Cardiol. 2018;3(6):532-40.

80. Mehra R, Benjamin EJ, Shahar E, Gottlieb DJ, Nawabit R, Kirchner HL, et al. Association of nocturnal arrhythmias with sleep-disordered breathing: the Sleep Heart Health Study. Am J Respir Crit Care Med. 2006;173(8):910-6.

81. Kanagala R, Murali NS, Friedman PA, Ammash NM, Gersh BJ, Ballman KV, et al. Obstructive sleep apnea and the recurrence of atrial fibrillation. Circulation. 2003;107(20):2589-94.

82. Caples SM, Mansukhani MP, Friedman PA, Somers VK. The impact of continuous positive airway pressure treatment on the recurrence of atrial fibrillation post cardioversion: a randomized controlled trial. Int J Cardiol. 2019;278:133-6.

83. Vazir A, Hastings PC, Dayer M, McIntyre HF, Henein MY, Poole-Wilson PA, et al. A high prevalence of sleep disordered breathing in men with mild symptomatic chronic heart failure due to left ventricular systolic dysfunction. Eur J Heart Fail. 2007;9(3):243-50. 
84. Orr JE, Malhotra A, Sands SA. Pathogenesis of central and complex sleep apnoea. Respirology.

2017;22(1):43-52.

85. Sands SA, Owens RL. Congestive heart failure and central sleep apnea. Sleep Med Clin. 2016;11(1):12742.

86. Savard J, Simard S, Blanchet J, Ivers H, Morin CM. Prevalence, clinical characteristics, and risk factors for insomnia in the context of breast cancer. Sleep. 2001;24(5):583-90.

87. Cimprich B. Pretreatment symptom distress in women newly diagnosed with breast cancer. Cancer Nurs. 1999;22(3):185-94 quiz 95.

88. Fiorentino L, Ancoli-Israel S. Insomnia and its treatment in women with breast cancer. Sleep Med Rev. 2006;10(6):419-29.

89. Davidson JR, MacLean AW, Brundage MD, Schulze K. Sleep disturbance in cancer patients. Soc Sci Med. 2002;54(9):1309-21.

90. Berger AM, Parker KP, Young-McCaughan S, Mallory GA, Barsevick AM, Beck SL, et al. Sleep wake disturbances in people with cancer and their caregivers: state of the science. Oncol Nurs Forum. 2005;32(6):E98126.

91. Qaseem A, Kansagara D, Forciea MA, Cooke M, Denberg TD, Clinical Guidelines Committee of the American College of P. Management of chronic insomnia disorder in adults: a clinical practice guideline from the American College of Physicians. Ann Intern Med. 2016.

92. Ritterband LM, Thorndike FP, Ingersoll KS, Lord HR, Gonder-Frederick L, Frederick C, et al. Effect of a webbased cognitive behavior therapy for insomnia intervention with 1-year follow-up: a randomized clinical trial. JAMA Psychiatry. 2017;74(1):68-75

Highlights a web-based approach to provide telemedicine care for patients with insomnia.

93. Ritterband LM, Bailey ET, Thorndike FP, Lord HR, Farrell-Carnahan L, Baum LD. Initial evaluation of an internet intervention to improve the sleep of cancer survivors with insomnia. Psychooncology. 2012;21:695-705.

94. Watson PL, Pandharipande P, Gehlbach BK, Thompson JL, Shintani AK, Dittus BS, et al. Atypical sleep in ventilated patients: empirical electroencephalography findings and the path toward revised ICU sleep scoring criteria. Crit Care Med. 2013;41(8):1958-67.

95. Gazendam JAC, Van Dongen HPA, Grant DA, Freedman NS, Zwaveling JH, Schwab RJ. Altered circadian rhythmicity in patients in the ICU. Chest. 2013;144(2):483-9.
96. Thille AW, Reynaud F, Marie D, Barrau S, Rousseau L, Rault C, et al. Impact of sleep alterations on weaning duration in mechanically ventilated patients: a prospective study. Eur Respir J. 2018;51(4).

97. Drouot X, Bridoux A, Thille AW, Roche-Campo F, Cordoba-Izquierdo A, Katsahian S, et al. Sleep continuity: a new metric to quantify disrupted hypnograms in non-sedated intensive care unit patients. Crit Care. 2014;18(6):628.

98. Sockalingam S, Parekh N, Bogoch II, Sun J, Mahtani R, Beach C, et al. Delirium in the postoperative cardiac patient: a review. J Card Surg. 2005;20(6):560-7.

99. Hollinger A, Ledergerber K, von Felten S, Sutter R, Ruegg S, Gantner L, et al. Comparison of propofol and dexmedetomidine infused overnight to treat hyperactive and mixed ICU delirium: a protocol for the Basel ProDex clinical trial. BMJ Open. 2017;7(7):e015783.

100. Devlin JW, Al-Qadhee NS, Skrobik Y. Pharmacologic prevention and treatment of delirium in critically ill and non-critically ill hospitalised patients: a review of data from prospective, randomised studies. Best Pract Res Clin Anaesthesiol. 2012;26(3):289-309.

101. Fitzgerald JM, Adamis D, Trzepacz PT, O'Regan N, Timmons S, Dunne C, et al. Delirium: a disturbance of circadian integrity? Med Hypotheses. 2013;81(4):568-76.

102. Park SY, Lee HB. Prevention and management of delirium in critically ill adult patients in the intensive care unit: a review based on the 2018 PADIS guidelines. Acute Crit Care. 2019;34(2):117-25.

103. Ibrahim MG, Bellomo R, Hart GK, Norman TR, Goldsmith D, Bates S, et al. A double-blind placebocontrolled randomised pilot study of nocturnal melatonin in tracheostomised patients. Crit Care Resusc. 2006;8(3):187-91.

104. Reade MC, O'Sullivan K, Bates S, et al. Dexmedetomidine vs. haloperidol in delirious, agitated, intubated patients: a randomised open-label trial. Crit Care. 2009;13:R75.

105. Lugaresi E, Provini F, Cortelli P. Agrypnia excitata. Sleep Med. 2011;12(Suppl 2):S3-10.

\section{Publisher's Note}

Springer Nature remains neutral with regard to jurisdictional claims in published maps and institutional affiliations. 